\title{
Validade externa da Escala de conforto para familiares de pessoas em estado crítico de saúde (ECONF) com Questionário sobre a saúde do paciente (PHQ)
}

\section{Felipe Ferreira Ribeiro de Souza1; Kátia Santana Freitas²; Bruna Luiza Pinheiro de Carvalho $^{3}$}

1. Bolsista PIBIC/CNPq, Graduando em Medicina, integrante do Núcleo Interdisciplinar de Pesquisas e Estudos em Saúde, Universidade Estadual de Feira de Santana, e-mail: felipeferreiraribeiro@ hotmail.com

2. Orientador, Professor Adjunto do Departamento de Saúde, Líder do Núcleo Interdisciplinar de Pesquisas e Estudos em Saúde (NIPES/DSAU/UEFS) e-mail: freitaskatia@ yahoo.com.br

3. Bolsista PIBIC/FAPESB, Graduanda em Psicologia, integrante do Núcleo Interdisciplinar de Pesquisas e Estudos em Saúde Universidade Estadual de Feira de Santana, e-mail: brunapsico13@ hotmail.com

PALAVRAS-CHAVE: depressão; escalas; família

\section{INTRODUÇÃO}

A constatação de uma doença e a hospitalização de um indivíduo adulto, que desempenha papéis bem definidos na família, podem gerar desequilíbrio em toda a estrutura familiar. Durante esta fase, é comum ocorrerem alterações de papéis e sentimentos de medo e insegurança que podem precipitar crises, das quais não podemos excluir transtornos psicopatológicos (VILA, ROSSI, 2002; GRAZZIANO, BIACHI, 2004).

Para adequada aferição de conforto e depressão nestes familiares, são utilizadas escalas validadas internacionalmente e no contexto brasileiro. Frente a esse panorama, Freitas (2012) construiu um instrumento que permite a avaliação do nível de conforto vivido por familiares no contexto da unidade de terapia intensiva, denominado Escala de Conforto para Familiares de Pessoas em Estado Crítico de Saúde (ECONF).

Assim, tendo em vista a importância de se utilizar instrumentos de medida validos, propõe-se o exame da validade da ECONF a partir de um critério externo. Nesse estudo a medida critério utilizada será o Questionário sobre a saúde do paciente-9 (Patient Health Questionnaire-PHQ-9), validado mundialmente, inclusive no Brasil (SPITZER, KROENKE, WILLIAMS, 1999; SPITZER et al, 2000; OSÓRIO et al, 2000). O PHQ-9 é considerado um instrumento útil tanto no diagnóstico quanto no prognóstico e acompanhamento da evolução da depressão no indivíduo, por demonstrar não só a presença de depressão, mas também a sua gravidade e resposta ao tratamento (KROENKE, SPITZER, 2002).

O escopo do trabalho foi buscar a validade externa da ECONF e sua correlação com o PHQ, devido à ausência de um instrumento padrão ouro para a medida do conforto. Além de verificar os níveis de conforto e depressão em familiares de pacientes internados em unidades de terapia intensiva, segundo o PHQ e a ECONF.

\section{METODOLOGIA}

Trata-se de um estudo transversal que foi realizado em duas Unidades de Terapia Intensiva (UTI) geral de um hospital público de grande porte, no município de Feira de Santana. O projeto obteve aprovação pela Comissão de Ética para Análise em Projetos de Pesquisa do Hospital Universitário Professor Edgar Santos, mediante o Parecer 078/09.

A população foi constituída de familiares de pessoas adultas internadas nas UTIs de um hospital público de Feira de Santana - BA, que atenderem aos seguintes critérios de inclusão: ter idade igual ou superior a 18 anos; ser a pessoa mais próxima do parente hospitalizado, que com ele convive e mantém relacionamento estreito; ter um familiar adulto internado na UTI com mais de $48 \mathrm{~h}$ de internação; ter realizado pelo menos uma visita ao parente. Foram inclusos 
no estudo aqueles indivíduos que atenderam os critérios de elegibilidade e aceitaram participar, no período de novembro de 2016 a julho de 2017.

Os dados sociodemográficos e clínicos, assim como a aplicação da ECONF, foram obtidos por meio entrevista. A ECONF é constituída de 55 itens, distribuídos em quatro dimensões: Segurança (20 itens), Suporte (21 itens), Interação consigo e com o cotidiano (08 itens), Interação familiar e ente (6 itens). A escala de medida é crescente, variando de 1 a 5 em cada uma das questões, ou seja, quanto maior o valor atribuído aos itens, maior é o grau de conforto. O PHQ-9 é uma das variações mais utilizadas do PHQ, sendo este o seu módulo isolado de depressão, o qual possui 9 questões. No PHQ, os critérios dizem respeito a alguns sinais e sintomas depressivos que o indivíduo sentiu nas últimas duas semanas. As opções de respostas variam de 0 a 3 , sendo que quanto maior a pontuação, maior a frequência de cada sintoma. As pontuações do teste variam entre 0 e 27 pontos, sendo que uma pontuação no intervalo de 0 a 4 é considerada como ausência de depressão, entre 5 e 9 pontos é considerada como uma depressão leve, entre 10 e 14 pontos, uma depressão moderada, entre 15 e 19 pontos, uma depressão moderadamente severa, e acima de 20 pontos, uma depressão severa (KROENKE, SPITZER, 2002).

Para a análise da validade de critério, o coeficiente de correlação pode mostrar a correspondência entre as duas escalas (MENEZES; 1998). Neste estudo, foi empregado o coeficiente de Correlação de Spearman, um método não paramétrico, utilizado devido aos testes de normalidade indicarem uma amostra anormal. O nível de significância estatística adotado foi de 5\%. Para análise das variáveis categóricas foi utilizada a estatística descritiva, como frequências absoluta e relativa. Para as variáveis quantitativas foram calculadas as medidas descritivas de centralidade (média, mediana e moda) e de dispersão (desvio- padrão).

\section{RESULTADOS E DISCUSSÃO}

Foram entrevistados familiares de 126 pacientes durante o período do estudo, com média de idade de 47 anos $( \pm 19,17)$, sendo a maioria dos pacientes do sexo masculino $(65,8 \%$; $\mathrm{n}=83)$, admitidos em proporção ligeriramente maior devido a causas cirúrgicas $(47,6 \% ; \mathrm{n}=$ 60) do que clínicas $(42,9 \% ; n=54)$. As principais causas de admissão na UTI foram o pósoperatório $(32,5 \% ; n=41)$, seguido de politrauma $(22,2 \% ; n=28)$.

Dos familiares adscritos no estudo, a maioria era do sexo feminino $(76,2 \% ; \mathrm{n}=96)$, católica $(50,8 \% ; n=64)$, com idade média de 37 anos $( \pm 11,1)$, casada ou com união consensual $(65,1 \% ; \mathrm{n}=82)$, com escolaridade de ensino médio $(53,2 \% ; \mathrm{n}=67)$ e em média com 10 anos $( \pm 3,7)$ de estudo. A maior parte tinha vínculo empregatício no momento $(50 \% ; n=44)$ e era residente de Feira de Santana - Bahia (54\%; $\mathrm{n}=68$ ). Quanto ao grau de parentesco com o paciente internado, os mais frequentes foram irmão(s), com $31 \%$ dos casos $(n=39)$, seguido de filho (a) $(24,6 \% ; n=31)$ e cônjuge $(19,8 \% ; n=25)$. A provável proximidade do paciente, característica contemplada na maior parte dos entrevistados por se tratarem de familiares de primeiro grau, sugere que os sentimentos expressos pelos sujeitos do estudo retratam o impacto da internação na vida dos familiares. Contudo, a maioria dos parentes entrevistados não morava com o familiar internado $(59,5 \% ; \mathrm{n}=75)$.

No que diz respeito à experiência com outros parentes internados em UTI ao ambiente de terapia intensiva, 34,1\% $(n=43)$ dos entrevistados disseram já ter tido. A média de visitas realizadas até o momento da entrevista foi de cerca de 5. Quanto ao Grupo de Apoio à Família (GAF), desenvolvido na sala de espera da UTI durante significativo período do estudo, 19,8\% $(n=25)$ dos entrevistados disseram ter presenciado ao menos 1 atividade do GAF. 
Quanto ao nível de conforto identificado após aplicação da ECONF, considerando-se a escala de respostas com pontuações que variam de 1 a 5 , sendo uma escala crescente, ou seja, quanto maior a pontuação, maior o nível de conforto relatado. O escore médio da ECONF foi de 3,83 ( $\pm 0,5)$. A pontuação geral da ECONF é classificada em três faixas, segundo Freitas (2012). São elas: pouco conforto $(<4,19)$; médio conforto $(4,20-4,59)$ e elevado conforto $(>4,6)$. Segundo estes valores, os familiares entrevistados possuíam pouco conforto diante da internação hospitalar do seu ente querido.

Quanto ao PHQ, escala que indica probabilidade de determinados graus de depressão, há a subdivisão em leve, moderada, moderadamente severa e severa, conforme maior for a pontuação (FOWLER et al, 2015; KROENKE \& SPITZER, 2002; SMARR \& KEEFER, 2011). Os resultados obtidos segundo esta divisão foram apresentados na Tabela 3. Outra forma de classificação a partir desta escala, indicada por diversos autores, é a de depressão maior ausente ou presente, a partir de pontuações $<10$ ou $\geq 10$, respectivamente. (BEARD et al, 2016; BERGEROT, LAROS, ARAUJO, 2014; KROENKE, SPTIZER \& WILLIANS, 2001; MILETTE et al, 2010; RATHORE et al, 2014). Considerando este quesito, verificou-se quase uma equivalência entre entrevistados que provavelmente possuem depressão maior $(47,6 \%$; $n$ $=60)$ e os que não possuem $(52,4 \% ; n=66)$.

Um dos fatores que podem estar relacionados às altas taxas de depressão, foi o estado o paciente no momento da realização da entrevista. No momento em que o familiar foi entrevistado, a maioria dos pacientes eram considerados graves porém estáveis pela equipe assistente da UTI (48\%; $\mathrm{n}=60)$. 24,8\% dos pacientes $(\mathrm{n}=31)$ foram considerados estáveis, ao passo em que sendo que $19,8 \%(\mathrm{n}=25)$ deles estavam graves e instáveis ou gravíssimos.

Para a análise da validade de critério da ECONF, inicialmente foi verificada a normalidade de distribuição por meio dos testes de Kolmogorov-Smirnov e Shapiro-Wilk, evidenciaram que, de maneira geral, a amostra não apresentou normalidade estatisticamente significante. Desta forma, optou-se pela realização de testes não paramétricos para a análise dos dados. Utilizando-se a correlação de Spearman para a análise da validade de critério da ECONF e do PHQ, obteve-se uma correlação negativa moderada $(r=-0,358)$ com significância estatística $(\mathrm{p}=0,000)$, visto que há relação inversamente proporcional entre a pontuação da escala ECONF e da PHQ. Ou seja, quanto maior a pontuação dos entrevistados na primeira escala, menor tende a ser a pontuação na segunda. Em outras palavras, familiares que relataram maior grau de conforto, apresentaram menores níveis de depressão, ao passo em que aqueles que mostraram maiores níveis de depressão tiveram menor grau de conforto.

\section{CONSIDERAÇÕES FINAIS}

A validade da ECONF foi reforçada ao evidenciar que familiares que apresentaram menores médias de conforto durante a internação do seu parente na UTI apresentam maiores escores para depressão. Este resultado contribui para o fortalecimento da Escala de conforto de familiares como medida válida, ao evidenciar que esta escala possui validade externa quando aplicada diante de outra escala bem estabelecida na literatura.

\section{REFERÊNCIAS}

BEARD C, HSU K.J.; RIFKIN L.S.; BUSCH A.B.; BJÖRGVINSSON T. Validation of the PHQ-9 in a psychiatric sample. J Affect Disord, v. 193, p.267-273, 2016. 
BERGEROT, C. D.; LAROS, J. A.; ARAUJO, T. C. C. F. Avaliação de ansiedade e depressão em pacientes oncológicos: comparação psicométrica. Psico-USF, Itatiba, v. 19, n. 2, p. 187197, 2014.

FOWLER J.C. et al. Early identification of treatment non-response utilizing the Patient Health Questionnaire (PHQ-9). J Psychiatr Res, v. 68, p. 114-119, 2015.

GRAZZIANO, E.S.; BIANCHI, E.R.F. Nível de ansiedade de clientes submetidos a cineangiocoronariografia e de seus acompanhantes. Rev Latinoam Enferm, v. 12, n. 2, p. 168174, 2004.

KROENKE, K.; SPITZER, R.L. The PHQ-9: A new depression and diagnostic severity measure. Psychiatric Annals, v. 32, n.9, p. 509-521, 2002.

KROENKE K.; SPITZER R.L.; WILLIAMS J.B.W. The PHQ-9. J Gen Intern Med, v.16, n. 9, p. 606-6013, 2001.

MENEZES, P.R. Validade e confiabilidade das escalas de avaliação em psiquiatria. Rev.Psiquiatr.Clín, São Paulo, v.25, n.5, p.241-246, 1998.

MILETTE K.; HUDSON M.; BARON M.; THOMBS B.D. Comparison of the PHQ-9 and CES-D depression scales in systemic sclerosis: internal consistency reliability, convergent validity and clinical correlates. Rheumatology, v.49, n.4, p. 789-796, 2010.

OSÓRIO, F. L. et al. Study of the discriminative validity of the PHQ-9 and PHQ-2 in a sample of Brazilian women in the context of primary health care. Perspectives in Psychiatric Care, v. 45, p. 216-227, 2009.

RATHORE J.S. et al. Validation of the Patient Health Questionnaire-9 (PHQ-9) for depression screening in adults with epilepsy. Epilepsy Behav, v. 37, p. 215-220, 2014.

SMARR K.L.; KEEFER A.L. Measures of depression and depressive symptoms: Beck Depression Inventory-II (BDI-II), Center for Epidemiologic Studies Depression Scale (CESD), Geriatric Depression Scale (GDS), Hospital Anxiety and Depression Scale (HADS), and Patient Health Questionna. Arthritis Care Res, v.63, n.11, p. 454-466, 2011.

SPITZER, R.L.; KROENKE, K.; WILLIAMS, J.B.W. Validation and utility of a self-report version of PRIME-MD: the PHQ PrimaryCare Study. JAMA, v. 282, p. 1737-1744, 1999.

SPITZER, R.L. et al. Validity and utility of the Patient Health Questionnaire in assessment of 3000 obstetrics-gynecologic patients. Am J ObstetGynecol, v. 183, p. 759-769, 2000.

VILA, V. S. C.; ROSSI, L. A. O significado cultural do cuidado humanizado em unidade de terapia intensiva: "muito falado e pouco vivido". Rev. Latino-Am. Enfermagem, Ribeirão Preto, v. 10, n. 2, p. 137-144, 2002. 